Relations industrielles

Industrial Relations

\title{
La gestion du stress
}

\section{Hans Selye et Pierre Turcotte}

Volume 31, numéro 4, 1976

URI : https://id.erudit.org/iderudit/028745ar

DOI : https://doi.org/10.7202/028745ar

Aller au sommaire du numéro

\section{Éditeur(s)}

Département des relations industrielles de l'Université Laval

ISSN

0034-379X (imprimé)

1703-8138 (numérique)

Découvrir la revue

Citer cet article

Selye, H. \& Turcotte, P. (1976). La gestion du stress. Relations industrielles / Industrial Relations, 31(4), 609-616. https://doi.org/10.7202/028745ar
Résumé de l'article

Les auteurs se demandent si l'état de turbulence observé dans le monde du travail n'est pas en fait une réponse non spécifique de la collectivité face à des exigences de plus en plus grandes pour l'industrie.
Tous droits réservés (C) Département des relations industrielles de l'Université Laval, 1976
Ce document est protégé par la loi sur le droit d'auteur. L'utilisation des services d'Érudit (y compris la reproduction) est assujettie à sa politique d'utilisation que vous pouvez consulter en ligne.

https://apropos.erudit.org/fr/usagers/politique-dutilisation/ 


\title{
La gestion du stress
}

\author{
Hans Selye \\ et \\ Pierre Turcotte
}

Les auteurs se demandent si l'état de turbulence observé dans le monde du travail n'est pas en fait une réponse non spécifique de la collectivité face à des exigences de plus en plus grandes pour l'industrie.

La société actuelle et plus particulièrement la société nord-américaine abonde en sources variées de stimuli stresseurs. L'entreprise a contribué dans une large mesure, au moyen des progrès technologiques dont elle est la principale instigatrice, à créer cet environnement tensiogène. Les nombreuses grèves et les revendications salariales constantes des travailleurs constitueraient plausiblement selon nous, une réponse de résistance à caractère catatoxique: lutte active contre les éléments stresseurs, en regard de la réponse à caractère syntoxique pouvant être définie comme un état de tolérance passive qui permet une sorte de symbiose ou coexistence pacifique avec les agresseurs. Les revendications précitées servant néanmoins tout au plus à compenser certaines frustrations accumulées, elles seraient le symptôme d'un mal difficile à déterminer. Si nous acceptons que le stress soit défini comme étant la réponse non spécifique que donne le corps à toute demande qui lui est faite, on peut se demander si cet état de turbulence dans le monde du travail, n'est pas en fait une réponse non spécifique de la collectivité face à des exigences de plus en plus grandes pour l'individu.

Même s'il est généralement admis que la présente génération de travailleurs est plus éduquée et plus critique que celles qui l'ont précédée, il n'en demeure pas moins que face aux demandes qui lui sont faites, elle doit rechercher une éthique de conduite; la génération actuelle doit, en effet, tenter de répondre à son tour à certaines questions devant préciser l'orientation de sa vie, de façon à réduire l'incertitude et par là le stress, l'incertitude étant généralement source de stress. Or, si l'on s'entend pour dire que le but ultime de la vie est de maintenir sa propre identité et de réaliser son potentiel, nous

SELYE, H., Dr., directeur de l'Institut de médecine et de chirurgie expérimentales de l'Université de Montréal. Président de l'Institut international du stress

TURCOTTE, P. C.A., Ph.D., professeur agrégé à la Faculté d'Administration de l'Université de Sherbrooke 
croyons que la possibilité de pouvoir se réaliser au travail en est d'autant plus importante, puisque l'homme passe près du tiers de sa vie à travailler. Cet espace de vie, plus que les autres, devrait dès lors être satisfaisant et enrichissant.

\section{PARTICIPATION ET STRESS}

Le besoin d'évoluer et de faire évoluer l'entreprise est devenu une des principales préoccupations du monde des affaires d'aujourd'hui. Pour l'entreprise actuelle, le style de gestion autoritaire est largement dépassé. Les valeurs sociales nouvelles, l'augmentation du niveau d'éducation et les demandes d'autonomie sans cesse croissantes de la part des individus laissent de moins en moins de place à des décisions unilatérales.

De plus, les cadres qui se plaignent fréquemment de ne pas disposer d'une autorité en proportion de leurs responsabilités soulèvent un problème d'influence. "L'individu qui n'a pas le sentiment de pouvoir influencer les évènements importants de sa vie professionnelle ou de sa carrière est une proie tout indiquée pour le stress ». ${ }^{1}$

Un nombre de plus en plus grand d'auteurs, de cadres et de dirigeants d'entreprises semblent croire que la formule de la participation au management peut être la solution permettant de mettre en accord les structures et mentalités dans l'entreprise avec l'évolution de la société. La participation pouvant ici être définie comme un style de gestion qui permet aux membres de l'entreprise à tous les niveaux, de prendre part à la prise de décision qui est directement reliée à leur action, en leur accordant le pouvoir concomitant et en leur déléguant les responsabilités qui s'y rattachent.

«La liberté d'entreprendre étant étroitement tributaire des institutions démocratiques, les hommes d'affaires doivent faire des efforts particulièrement vigilants pour s'adapter aux circonstances présentes. La voie du juste milieu est souvent plus étroite qu'on est porté à le croire. Dans le contexte actuel, elle passe fatalement par la participation et par la concertation; c'est-à-dire, par une discipline sévère qui exige qu'on fasse une plus juste place à l'homme dans le travail quotidien aussi bien que dans les démarches collectives $»{ }^{2}$

1 Bulletin mensuel, Banque Canadienne Nationale, Vol. 52, No. 11, Novembre 1976.

2 TURCOTTE, P. R., La participation au management, étude présentée par le Centre des dirigeants d'entreprise, Montréal, 1976. 
Devant cette tendance de plus en plus prononcée de recourir à la participation comme solution aux problèmes de l'entreprise, on peut se demander quel sera l'effet de ce nouveau style de gestion en regard du stress qui lui sera apparenté. Cet article tentera de démontrer que la participation au management est susceptible de réduire le stress désagréable relié à la situation de travail.

Tout stress n'est toutefois pas nécessairement à éviter. La présence de stress nocif, état que l'on appelle détresse, est dans le cadre de l'entreprise, souvent reliée à la prise de décision unilatérale: égoisme générateur de stress négatif. Le stress engendré par un niveau de motivation adéquat est à maintenir si l'on admet l'existence d'un niveau optimal de stress. Un code de conduite personnelle qui se traduit par le fait de se mériter la bonne volonté et l'estime d'autrui est fondamental dans le maintien du stress nocif à son plus bas niveau possible. «Le stress engendré par les conflits, la haine et la frustration serait évité, et nous atteindrions à la joie si nous accordions plus d'attention à la compréhension des bases naturelles de la motivation et de la conduite. Celui qui, dans la vie de tous les jours aura appris à se conformer au précepte 'mérite l'amour de ton prochain' ne sera jamais déçu». ${ }^{3}$ Parallèlement, l'entreprise peut plausiblement tenter de maintenir le stress à son niveau souhaitable par l'élaboration d'un code de conduite (réducteur de stress) qui serait relié à une idéologie participative.

\section{MOTIVATION ET STRESS}

L'approche, à partir de la motivation, est très courante dans les études se rapportant au travail. La plupart des auteurs sont d'accord pour admettre qu'une absence de motivation souvent inhérente aux tâches routinières, est une des sources les plus fréquentes de dévalorisation chez l'individu, ces tâches conduisant souvent à l'ennui, source fréquente de détresse. De même, l'homme a besoin d'harmonie et ne peut supporter constamment la critique, c'est ce qui, plus que tout autre agent de stress, rend le travail malsain et nuisible, bloque les efforts créatifs de l'entreprise et étouffe les efforts de réalisation de l'individu. Des salaires plus élevés et des bénéfices marginaux accrus servent dans de nombreux cas de compensation à d'autres besoins ne pouvant être satisfaits à l'intérieur de l'organisation.

Même si n'importe quelle occupation peut permettre de retirer un minimum de sentiment d'accomplissement, le travail devrait être en

3 SELYE, Hans, Stress sans détresse, Les éditions La Presse, Montréal, 1974. 
lui-même une source de satisfaction intrinsèque des besoins fondamentaux de l'homme. Les membres qui ont su se réaliser à l'intérieur de l'entreprise seront moins exigeants en termes de récompenses extrinsèques.

Ceux d'entre nous qui recueillent bienveillance et affection n'ont pas le même besoin d'argent que d'autres, car une large part de ce qu'ils peuvent désirer leur est offert. Pour que l'individu puisse se réaliser, il faut que l'entreprise lui fournisse les opportunités adéquates, sans quoi, il cherchera ailleurs des substituts; la participation d'après ses compétences propres ou près des centres de réalisation peut s'avérer une source de satisfaction intrinsèque.

Car en fait, se réaliser, c'est une façon d'exprimer ouvertement son identité et de faire valoir son potentiel, ce qui permet à l'individu une authenticité dans laquelle le stress désagréable est à son minimum. De plus, l'activité couronnée de succès, peu importe son intensité, laisse peu de cicatrices. Elle provoque peu de stress et pas de détresse. Elle apporte, au contraire, le sentiment exaltant de force propre à la jeunesse, même à un âge avancé.

\section{DÉVELOPPEMENT ORGANISATIONNEL}

Parmi les gens assurant les emplois les plus courants dans la société actuelle, aux échelons inférieurs et moyens des affaires, dans l'industrie, l'agriculture et les services publics (depuis l'homme à tout faire, jusqu'à l'administrateur ou le fonctionnaire avec des responsabilités limitées), l'une des plus grandes causes de détresse est le mécontentement que leur apporte leur vie, à savoir, un manque de considération pour leur travail. Il ne faut pourtant pas que les individus évitent le travail, mais qu'ils recherchent celui qui serait un plaisir; ils en réduisent d'autant leur détresse. Comme on le sait, au moment de son engagement, le travailleur a souvent plus ou moins choisi son travail et il a vraisemblablement accepté la seule tâche offerte. Ceci est d'autant plus vrai, si ce dernier était en chômage depuis un certain temps avant son engagement.

Le travail est une nécessité biologique. Il est bien certain qu'idéalement, un choix de carrière répondant totalement aux aspirations de l'individu serait souhaitable. Cependant, la réalité est nettement éloignée de cette utopie; l'individu doit souvent s'accommoder d'une tâche génératrice d'un certain niveau d'ennui et d'un certain plafond quant à ses ambitions de réalisation, phénomènes tous deux porteurs de stress. 
Nous croyons que penser en terme de participation, c'est penser en terme de tâches intégrées. Il est possiblement inutile de vouloir jouer sur le climat de travail, si à la base, la tâche est ingrate ou mal organisée. Il doit y avoir une ambition d'accomplir quelque chose qui satisfait en profondeur, pour réduire le stress. Il ne faut pas donner au travailleur la sécurité, mais bien l'occasion à celui-ci de se réaliser d'après ses talents ; ce qui va le sécuriser et par conséquent, réduire sa détresse.

L'ennui, résultant des tâches routinières, peut être circonscrit par des politiques de rotation et d'enrichissement des tâches, qui, tout en accroissant les connaissances et la capacité de prendre des décisions, contribuent à la satisfaction individuelle. Une action en développement organisationnel qui débouche sur tout l'organisation permet à l'individu d'entrevoir des possibilités nouvelles de réalisation, l'homme ne connaissant pas de limites quand il travaille à son propre perfectionnement.

Dans ce contexte, croître avec sa tâche signifie que l'individu puisse devenir davantage maitre de son sort et puisse utiliser une part plus large de son potentiel humain qu'est, entre autre, sa capacité de décider. La décision prise par un autre est facilement contestable alors qu'il en est différent pour une décision qu'on a soi-même prise. La participation à la prise de décision ne devrait idéalement se faire que dans un climat de coopération fertile en stress positif tout en permettant de réduire à son minimum le stress négatif.

Comme l'indique Michel Crozier: «participer, c'est perdre de sa liberté, c'est perdre la situation favorable du critique confortablement à l'abri... ${ }^{4}$ En fait, la participation au management qui fait dorénavant du dirigeant une personne-ressource, fait fortement appel à la capacité d'adaptation de celui-ci. Participer, c'est en effet accepter une certaine part d'insécurité: le cadre qui délègue de son pouvoir réduit sa propre sphère d'action et doit prévoir un emploi différent de son temps.

Un programme de développement des échelons inférieurs doit prévoir une formation technique de façon à rendre les membres plus compétents dans la prise de décision, et une formation psychologique afin de mieux les disposer à coopérer et à prendre des décisions, et dès lors à réduire le stress désagréable relié aux incertitudes ainsi créées.

L'apprentissage de ces nouveaux rôles en est un qui suscitera, à court terme, nombre de tensions chez le dirigeant à un point tel que celui-ci souhaitera, à l'occasion, se retrouver dans une structure plus 
autoritaire comportant moins d'incertitude. Par contre, le long terme de la structure participative, tel qu'il a été développé précédemment quant à ses possibilités de réalisation est plus prometteur en termes d'absence de stress que la structure traditionnelle.

POUVOIR

La participation implique très souvent la co-décision et par conséquent, un nouveau partage du pouvoir, une forme d'associationisme générateur d'égoïsme altruiste, pouvant conduire à une coopération paisible et satisfaisante pour tous. Comme on l'a vu, l'usage unilatéral du pouvoir est par contre souvent la source d'un égoïsme culpabilisant et anxiogène. C'est l'utilisation de ce pouvoir unilatéral qui est fréquemment à la base d'un antagonisme généralisé dans les relations de travail. Nous vivons dans un système «win-lose». Ce qui est gagné par le patron est perdu par l'employé et se traduit par une compétition interne. Il ne s'agit pas d'une indifférence mutuelle qui permet la coexistence (attitude syntoxique), mais bien d'une confrontation ouverte (catatoxique).

La participation pourrait permettre de transformer ce système «win-lose» en un système «win-win», où il est possible de réconcilier les objectifs organisationnels avec les objectifs individuels. Si ne pas avoir de but personnel est générateur de stress désagréable, nous pouvons croire que l'entreprise qui permet à ses employés l'introjection de ses objectifs organisationnels fournira à ces derniers un but générateur de stress agréable.

\section{GROUPE DE TÂCHES}

Les techniques du «team building» peuvent être utiles à ceux qui privilégient la participation. Ces techniques permettent aux groupes de tâches de développer des normes et rôles qui sont propres à leurs membres et qui répondent aux besoins spécifiques d'une tâche. Si les dirigeants d'entreprise croient en la participation, ils admettront que dans des situations appropriées, les individus sont responsables; il naîtra alors un sentiment de confiance réciproque essentiel à la participation.

Si les membres d'une organisation adhèrent aux objectifs de celleci, il s'ensuivra un phénomène de symbiose, c'est-à-dire une plus grande interdépendance entre les individus qui poursuivent les mêmes objectifs. 
Le développement d'un système d'aide mutuelle entre les parties d'un organisme affaiblit le stress interne, c'est-à-dire les frictions et permet une coexistence harmonieuse entre toutes les parties du corps. Le groupe social, de son côté, créera des pressions à la conformité qui réduiront de ce fait la nécessité d'une surveillance étroite, elle-même propice au stress négatif. Le «team building» doit conduire à une réduction du contrôle direct et à la prise des responsabilités, en développant le sens de la communauté. Les individus ont tous des éthiques de conduite qui leur sont propres. Au niveau du contrôle global, il s'agira de dégager les éléments communs et de les intégrer à l'organisation. En fait, nous croyons que la nature même des groupes de tâches composés de membres hétérogènes contribue nécessairement à renforcer le sentiment d'interdépendance nécessaire à l'atteinte des objectifs et conduit à l'émergence d'une forme d'égoïsme altruiste. Les ambitions propres des individus se heurtent moins à celles des autres et il y a alors réduction du stress interpersonnel.

Si l'on engendre et respire l'amour, l'on ne peut pas être attaqué et alors il y a maintien de l'équilibre homéostatique. Alors, naît un sentiment de sécurité. Le dirigeant qui a confiance en la capacité de l'être humain va engendrer l'amour (gratitude-bienveillance) et ses subalternes lui seront redevables et accepteront éventuellement de ce fait des responsabilités accrues. Il est relativement aisé de rechercher l'égoïsme altruiste qui essaye d'aider autrui dans le dessein égoïste de s'en faire un obligé, sur lequel on est en droit, à son tour de compter. La considération apportée au travailleur dans cette quête d'égoïsme altruiste, en plus d'aller dans le sens des objectifs organisationnels, contribuera en fait à réduire la détresse de ce dernier.

\section{CONCLUSION}

L'État tend, de plus en plus, à prendre des responsabilités qui étaient autrefois réservées à l'individu, que ce soit dans les domaines scolaires, de la santé ou de la sécurité sociale. De même en est-il des entreprises, qui ne sachant pas prendre leurs responsabilités à temps, ont été devancées par l'État. Dans un contexte semblable, les individus et les entreprises ont perdu leur sens des responsabilités et une part de leur autonomie et ils participent à peine aux prises de décisions collectives; et l'on voudrait dans le cadre de la participation au management que les membres d'une entreprise soient autonomes, responsables et capables d'une certaine auto-direction! Il va de soi que l'on est conséquemment en face d'un objectif de taille qui demandera 
la mise en commun de bon nombre d'efforts tant collectifs qu'individuels.

Tel que nous l'avons mentionné précédemment, les décisions prises unilatéralement ne sont pas une source de satisfaction pour ceux qui doivent les subir et sont même porteuses de détresse. La participation nous semble, pour sa part, intimement reliée au stress (ou plus exactement en fait, à la diminution du stress) et doit conduire inévitablement à une certaine forme d'égoïsme altruiste qui ne soit pas anxiogène et qui ne génère pas de sentiments de culpabilité.

Nous prétendons qu'une idéologie de participation bien comprise (car il faut éviter de penser en termes de recettes programmables, il faut parler d'esprit, de climat, d'attitudes) saura nécessairement faire naître chez chaque membre de l'organisation, des sentiments d'ouverture sur autrui ayant pour fondement la gratitude et la bienveillance, au lieu des sentiments de haine et de vengeance tels que vécus dans le cadre actuel de l'antagonisme des relations ouvrières.

La participation au management sera, de toute évidence, une solution inévitable aux problèmes que nous vivons présentement. Il ne faut pas attendre que la base revendique cette participation qui lui est légitime, comme ce fut le cas à l'Université Laval: "ces hommes et ces femmes à qui l'on confie le soin d'enseigner et de faire rayonner leur université par leurs travaux et leurs écrits, on ne leur reconnaît ni le sens des responsabilités, ni un respect suffisant de leur institution pour leur permettre de prendre en groupe des décisions qui les concernent directement... $\gg^{5}$ ou encore attendre que le gouvernement l'impose (hétérostasie), mais bien que les dirigeants prennent les devants et instaurent eux-mêmes la participation qui assurera la survie de leur entreprise. Nous n'aurons plus alors une participation à la détresse, mais plutôt une saine gestion du stress.

Il semble que tous souhaitent une meilleure harmonie entre les entreprises et la collectivité. Ceci est possible si ces dernières développent, comme c'est le cas sur le plan individuel, un code d'éthique ou de conduite favorisant de meilleures relations. En effet, si les entreprises développent une forme d'égoïsme altruiste en engendrant la gratitude et la bienveillance, elles ne pourront pas être attaquées et se feront des membres de la société des obligés, ce qui permettra éventuellement le maintien de leur homéostasie. 